\title{
In vitro performance in cotton plants with different genetic backgrounds: the case of Gossypium hirsutum in Mexico, and its implications for germplasm conservation
}

\author{
Alejandra Hernández-Terán ${ }^{1,2}$, Ana Wegier ${ }^{3}$, Mariana Benítez ${ }^{1,4}$, Rafael Lira ${ }^{5}$, Tania Gabriela Sosa Fuentes ${ }^{3}$, Ana \\ E. Escalante ${ }^{\text {Corresp. } 1}$ \\ ${ }^{1}$ Instituto de Ecología, Laboratorio Nacional de Ciencias de la Sostenibilidad, Universidad Nacional Autónoma de México, Mexico City, Mexico \\ 2 Programa de Doctorado en Ciencias Biomédicas, Universidad Nacional Autónoma de México, Mexico City, Mexico \\ 3 Instituto de Biología, Jardín Botánico, Universidad Nacional Autónoma de México, Mexico City, Mexico \\ ${ }^{4}$ Centro de Ciencias de la Complejidad, Universidad Nacional Autónoma de México, Mexico City, Mexico \\ 5 Facultad de Estudios Superiores Iztacala, Universidad Nacional Autónoma de México, Los Reyes, Mexico \\ Corresponding Author: Ana E. Escalante \\ Email address: aescalante@iecologia.unam.mx
}

One of the best ex situ conservation strategies for wild germplasm is in vitro conservation of genetic banks. The success of in vitro conservation relies heavily on the micropropagation or performance of the species of interest. In the context of global change, crop production challenges and climate change, we face a reality of intensified crop production strategies, including genetic engineering, which can negatively impact biodiversity conservation. However, the possible consequences of transgene presence for the in vitro performance of populations and its implications for biodiversity conservation are poorly documented. In this study we analyzed experimental evidence of the potential effects of transgene presence on the in vitro performance of Gossypium hirsutum L. populations, representing the Mexican genetic diversity of the species, and reflect on the implications of such presence for ex situ genetic conservation of the natural variation of the species. We followed an experimental in vitro performance approach, in which we included individuals from different wild cotton populations as well as individuals from domesticated populations, in order to differentiate the effects of domestication traits dragged into the wild germplasm pool via gene flow from the effects of transgene presence. We evaluated the in vitro performance of five traits related to plant establishment $(\mathrm{N}=300)$ : propagation rate, leaf production rate, height increase rate, microbial growth and root development. Then we conducted statistical tests (PERMANOVA, Wilcoxon post-hoc tests, and NMDS multivariate analyses) to evaluate the differences in the in vitro performance of the studied populations. Although direct causality of the transgenes to observed phenotypes requires strict control of genotypes, the overall results suggest detrimental consequences for the in vitro culture performance of wild cotton Peer) reviewing PDF | (2018:11:33125:3:0:NEW 17 Apr 2019) 
populations in the presence of transgenes. This provides experimental, statistically sound evidence to support the implementation of transgene screening of plants to reduce time and economic costs in in vitro establishment, thus contributing to the overarching goal of germplasm conservation for future adaptation. 


\section{In vitro performance in cotton plants with different genetic}

\section{2 backgrounds: the case of Gossypium hirsutum in Mexico, and its}

\section{3 implications for germplasm conservation}

4 Alejandra Hernández-Terán ${ }^{1,2}$, Ana Wegier ${ }^{3}$, Mariana Benítez ${ }^{1,4}$, Rafael Lira ${ }^{5}$, Tania Gabriela

5 Sosa Fuentes ${ }^{3}$, Ana E. Escalante ${ }^{1 *}$

6

$7{ }^{1}$ Instituto de Ecología, Laboratorio Nacional de Ciencias de la Sostenibilidad, Universidad Nacional 8 Autónoma de México, Mexico City, Mexico

$9{ }^{2}$ Programa de Doctorado en Ciencias Biomédicas, Universidad Nacional Autónoma de México, Mexico 10 City, Mexico

$11{ }^{3}$ Instituto de Biología, Jardín Botánico, Universidad Nacional Autónoma de México, Mexico City, 12 Mexico

$13{ }^{4}$ Centro de Ciencias de la Complejidad, Universidad Nacional Autónoma de México, Mexico City, 14 Mexico

$15{ }^{5}$ Facultad de Estudios Superiores Iztacala, Universidad Nacional Autónoma de México, Mexico City, 16 Mexico

*Corresponding author: aescalante@iecologia.unam.mx 


\section{Abstract}

24 One of the best ex situ conservation strategies for wild germplasm is in vitro conservation of 25 genetic banks. The success of in vitro conservation relies heavily on the micropropagation or 26 performance of the species of interest. In the context of global change, crop production 27 challenges and climate change, we face a reality of intensified crop production strategies, 28 including genetic engineering, which can negatively impact biodiversity conservation. However, 29 the possible consequences of transgene presence for the in vitro performance of populations and 30 its implications for biodiversity conservation are poorly documented. In this study we analyzed experimental evidence of the potential effects of transgene presence on the in vitro performance of Gossypium hirsutum L. populations, representing the Mexican genetic diversity of the species, and reflect on the implications of such presence for ex situ genetic conservation of the natural variation of the species. We followed an experimental in vitro performance approach, in which we included individuals from different wild cotton populations as well as individuals from domesticated populations, in order to differentiate the effects of domestication traits dragged into

37 the wild germplasm pool via gene flow from the effects of transgene presence. We evaluated the in vitro performance of five traits related to plant establishment $(\mathrm{N}=300)$ : propagation rate, leaf production rate, height increase rate, microbial growth and root development. Then we conducted statistical tests (PERMANOVA, Wilcoxon post-hoc tests, and NMDS multivariate analyses) to evaluate the differences in the in vitro performance of the studied populations. Although direct causality of the transgenes to observed phenotypes requires strict control of genotypes, the overall results suggest detrimental consequences for the in vitro culture

44 performance of wild cotton populations in the presence of transgenes. This provides 45 experimental, statistically sound evidence to support the implementation of transgene screening 
46 of plants to reduce time and economic costs in in vitro establishment, thus contributing to the 47 overarching goal of germplasm conservation for future adaptation. 


\section{Introduction}

49 Interest in plant germplasm conservation addresses the need to preserve a diverse genetic pool, 50 thus providing options for future decision-making (Rockstrom et al., 2014). Such options must

51 include genetic and phenotypic diversity to face current and future challenges in crop production.

52 The conservation of these variants can help in developing or finding solutions to disease,

53 changing environments, and low yields, among others, and is necessary for safeguarding 54 biodiversity and cultural identity (Hawkes, 1977; Plucknett et al., 1983; Hajjar \& Hodgkin, 55 2007). In most crops, the largest genetic variation exists in the Crop Wild Relatives (CWR) 56 found in the centers of origin of the species (Hawkes, 1977). Accordingly, research groups in 57 food security identified CWR as a target group for conservation (Harlan, 1965; Hunter \&

58 Heywood, 2011; Castañeda-Álvarez et al., 2016). Due to the importance of CWR for 59 conservation, international agreements have made the in situ and ex situ preservation of their genetic diversity one of their goals (Aichi Target 13) (Leadley et al., 2014).

Plant tissue culture methods represent a robust approach for many purposes, from being a tool designed to pursue a variety of basic research questions to helping ex situ preservation of 63 genetic diversity (Engelmann, 1991; Gosal \& Kang, 2012). The most successful example of 64 tissue culture in commercial and conservation applications is micropropagation: the propagation 65 of plants from small parts under in vitro conditions. The success of micropropagation in tissue 66 culture is due to ease of having multiple genetic clones from different geographic locations, thus

67 lowering the risk of loss of such genotypes (Kumar \& Reddy, 2011; Rajasekharan \& Sahijram, 68 2015).

Despite the great advantages of micropropagation for in vitro conservation of plant 70 species, different factors can compromise its success, such as culture medium composition, 
71 environmental conditions and genotype, among others (Li et al., 2002; Tyagi et al., 2004; Kumar

$72 \&$ Reddy, 2010). In fact, it has been shown that different cultivars of the same species can have

73 different in vitro performance or success (Gubis et al., 2003; Pathi \& Tuteja, 2013). This reveals

74 the sensitivity of in vitro culture to even small genetic variation. In the past few decades, the use

75 of Genetically Modified Organisms (GMO) has become extensive (ISAAA, 2017) and a source

76 of new genetic variation even in countries that are considered centers of origin of important

77 crops (Lu, 2008). In some cases, the release of GMOs in areas with CWR or with other crops and

78 weeds, has caused gene flow events across populations of different economically important

79 cultivars, such as maize, cotton, papaya, bent grass, alfalfa and canola (Quist \& Chapela, 2001;

80 Warwick et al., 2008; Piñeyro-Nelson et al., 2009; Wegier et al., 2011; Greene et al., 2015;

81 Manshardt et al., 2016). Thus, given the extensive use of GMO technologies in economically

82 important cultivars, it becomes relevant to analyze all evidence related to the effects of this

83 introduced variation on the in vitro culture germplasm conservation efforts.

Mexico is the center of origin for cotton (Gossypium hirsutum L.) (Ulloa et al., 2006;

Burgeff et al., 2014), and its metapopulations have been found on the coasts of the country, while

86 extensive cultivars can be observed in the northern states, and backyard/home garden plants and

87 native varieties have been reported in the southeastern states (Velázquez-López et al., 2018).

88 Previous studies have described the genetic diversity of the Mexican metapopulations, including

89 the presence of transgenes in some of them (Wegier et al., 2011), suggesting gene flow

90 associated with specific transformation events (i.e. transgene introduction) from extensive

91 cultivars to metapopulations. Ellstrand (2018) has posed the hypothesis that the majority of

92 Mexican cotton metapopulations do not correspond with wild relatives of cotton (truly wild), but

93 are instead a mix of escaped cultivar individuals that have evolved in wild conditions (weedy- 
94 wild). Nonetheless, even if the cotton metapopulations are weedy-wild relatives, they are part of 95 the primary genetic pool (Heywood et al., 2007) and are thus of conservation interest due to their 96 genetic diversity (Ellstrand, 2018). Phenotypic consequences of genetic flow (including 97 transgene flow) into wild and domesticated lines in other species have been suggested in 98 previous studies (Hernández-Terán et al., 2017); therefore, in vitro culture performance could 99 also be affected, in principle, by genetic modification. This could have important consequences 100 for the success of germplasm conservation strategies.

101 In the present study, and given the genetic diversity of G. hirsutum in Mexico, we 102 analyzed experimental evidence of the effects of transgene presence on the in vitro performance 103 of representative population clones of G. hirsutum diversity, and reflect on the implications of 104 such effects for ex situ genetic conservation. For these reasons, and given that transgene presence 105 in cotton metapopulations (hereafter wild germplasm) can be directly attributed to gene flow 106 from domesticated populations, we included individuals from domesticated populations in our 107 comparisons in order to differentiate the effect of domestication traits dragged into the wild 108 germplasm pool via gene flow from the mere presence of transgenes. Thus, we hypothesize that 109 i) given that transgenes are directed to specific traits (e.g. defense and herbicide tolerance), 110 which are not related to in vitro performance, we will not find differences in such performance

111 between populations with and without transgenes, and ii) the only differences to be found in the 112 in vitro performance will be those associated with the domestication process, between wild and 113 domesticated populations.

\section{Materials and methods}

\section{Experimental design}


116 To evaluate the potential effects of transgene presence on the in vitro culture performance of

117 wild cotton plants and its consequences for germplasm conservation efforts, we conducted a

118 systematic analysis of the performance of specific traits of an in vitro germplasm collection of

119 cotton plants. We included a representative sample of the genetic diversity of wild population

120 plants with $\left(\mathrm{W}_{\mathrm{T}}\right)$ and without $(\mathrm{W})$ transgenes (i.e. no isogenic lines), to test the effect of

121 transgenes on the in vitro performance of metapopulation variants. Given the interest of this

122 study for conservation strategies, we intentionally look for diversity of the genetic background of

123 the analyzed clones, in other words population level diversity. In addition, and as a preliminary

124 attempt to distinguish the effects attributable to transgenes from the effects attributable to flow

125 from domesticated or cultivar populations into wild populations, we included domesticated

126 plants with $\left(\mathrm{D}_{\mathrm{T}}\right)$ and without $(\mathrm{D})$ transgenes in the experiment and analyses.

\section{Germplasm collection}

128 In order to have a germplasm collection that is representative of the genetic diversity of wild 129 cotton populations in Mexico, we collected seeds from individual plants in populations spanning 130 its natural distribution or in metapopulations (Wegier et al., 2011). Ten seeds of each individual 131 plant in the collection were germinated in prepared substrate (Peat Moss, agrolite, vermiculite 132 (3:1:1) and $50 \mathrm{~g}$ of slow-release Osmocote fertilizer (14N-14P-14K, [Scott's, Marysville, Ohio]), 133 in a greenhouse under controlled conditions $\left(25 \pm 5^{\circ} \mathrm{C}\right)$. Once the seedlings emerged, the apex 134 and the first three axillary buds were explanted to start the in vitro culture. We also included 135 germplasm of domesticated plant individuals from farmer's markets in Mexico City as 136 representatives of the domesticated cotton populations. 
139 Establishment. Once disinfected (supplementary material, appendix S1), the axillary buds were

140 transferred to culture tubes containing $6 \mathrm{ml}$ of MS basal medium (Murashige \& Skoog, 1962).

141 Each tube was sealed with Parafilm M (Bemis, USA) to prevent contamination. The culture

142 tubes were incubated in a growth room at $24^{\circ} \mathrm{C}$ for a 12 -hours photoperiod.

143 Propagation. Propagation was initiated with individual explants that reached $8 \mathrm{~cm}$ height or the

144 height of the culture tube, or when the initial culture exhausted the culture medium. Propagation

145 consisted in removing the explants from the culture tubes, cutting each of the new axillary buds

146 and planting them in a culture tube with fresh medium. The process was performed under sterile

147 conditions in a laminar-flow hood (ThermoFisher, Massachusetts, USA). For more information,

148 see supplementary material (Appendix S1).

149 Detection of transgenes in the germplasm collection

150 To characterize the populations under evaluation (i.e. Wild (W) and Domesticated (D)), we

151 looked for two constructions of lepidopteran resistance and one of herbicide tolerance

152 (CrylAb/Ac, Cry2Ab and CP4EPSPS) in all individuals of the collection. This allowed us to

153 detect 23 of the 33 transgenic cotton events released in Mexico (ISAAA, 2018). For the wild

154 cotton populations $\left(\mathrm{W}\right.$ and $\mathrm{W}_{\mathrm{T}}$ ) we carried out two independent tests to verify the presence of the

155 genetic events: enzyme-linked immunoabsorbent assay (ELISA), and sequencing of Polymerase

156 Chain Reaction (PCR) products. For the domesticated cotton populations ( $\mathrm{D}$ and $\mathrm{D}_{\mathrm{T}}$ ), transgenic

157 events were verified only with a PCR-sequencing assay. The ELISA tests were performed in

158 duplicate using the following kits: Bt-Cry1Ab/1Ac ELISA Kit, Bt-Cry2A ELISA Kit, and 159 Roundup Ready ELISA Kit (Agdia, Elkhart, Indiana, USA). The results were read in a 160 MultiskanFC Microplate Photometer (ThermoFisher Scientific, Massachusetts, USA). We

161 considered a sample to be positive only when its absorbance was equal to or above three standard 
162 deviations from the average intensity of all negative controls and blank samples. In all ELISA

163 plates a blank sample (extraction buffer), a negative, and a positive control provided in each 164 detection kit were included. ELISA results are available as supplementary material 165 (ELISA_results.xslx).

166 For the PCR assays, DNA extraction was performed in duplicate for each individual, following 167 the DNA Miniprep CTAB method reported in Wegier and collaborators (2011). The quality and 168 concentration of the DNA were analyzed in a NanoDrop 2000 (ThermoFisher Scientific, 169 Massachusetts, USA). The PCR assay was performed with the primers Cry1 Ab/Ac (F 170 5'ACCGGTTACACTCCCATCGA 3', R 5'CAGCACCTGGCACGAACT 3'), Cry2Ab (F 171 5'CAGCGGCGCCAACTCTACG 3'

172 , R 5'TGAACGGCGATGCACCAATGTC 3'), and CP4EPSPS (F 173 5'GCATGCTTCACGGTGCAA 3', R 5'TGAAGGACCGGTGGGAGAT 3')

174 from Eurofins Scientific (Brussels, Belgium). The assay was carried out according to the 175 references provided in Appendix S3. Subsequently, the amplicons result of the PCR assay were 176 verified by Sanger sequencing. The sequencing was done in the Laboratorio of Biología 177 Molecular in the Instituto de Biología, UNAM. Raw sequences are available in GenBank 178 platform (accession \#MK089921-30; Appendix S5).

Data collection 
183 To evaluate the potential consequences of transgene presence for the in vitro performance of

184 cotton populations, we measured different traits of individuals in our germplasm collection. All

185 data analyzed is included as supplementary material (Supplementary_dataset.xlsx).

186 During the establishment of the in vitro germplasm collection we documented differences in 187 propagation success in a period of two years that included a total of 4,377 axillary buds 188 (corresponding to 74 individual original plants, 27 with transgenes and 47 without transgenes). 189 From this original sample, we randomly selected 20 individuals (five wild with transgenes $\left(\mathrm{W}_{\mathrm{T}}\right.$ ), 190 five wild without transgenes (W), five domesticated without transgenes (D) and five 191 domesticated with transgenes $\left.\left(\mathrm{D}_{\mathrm{T}}\right)\right)$, and 15 replicates per individual $(\mathrm{N}=300)$ to evaluate in vitro 192 performance of four phenotypic traits (leaf rate, height rate, microbial growth, and root 193 development). This collection was intended to be a fair representation of the wild cotton 194 metapopulations genetic backgrounds diversity, since it includes five out of the eight populations 195 reported in Mexico (Wegier et al., 2011) plus ten individuals from the domesticated genetic 196 background. Data for these traits were collected weekly during a four-month period. As 197 mentioned above, all the experiments were conducted in a growth room at $24{ }^{\circ} \mathrm{C}$ under a 12 198 hours photoperiod. A detailed scheme of the experimental design is available in the 199 supplementary material (Appendix S2).

200 Specifics for the evaluated traits are:

201 (i) propagation rate, calculated as the number of buds derived from a single individual 202 203 204 every two weeks during two years of continual propagation, or the slope of the linear regression model using the $l m$ function in $R$ (no. buds $V s$. time) $\left(\mathrm{W}_{\mathrm{T}} \mathrm{N}=1800\right.$, W $\mathrm{N}=2577, \mathrm{D} N=490, \mathrm{D}_{\mathrm{T}} \mathrm{N}=633$ ); 
205 206

207 208

209

210

211

212

213

214

215

216

217

218

219

220

221

222

223

22

224 To determine if there are statistical differences among experimental groups for all the traits 226 (PERMANOVA) (Legendre \& Anderson, 1999) based on 1000 permutations using adonis 227 function in vegan $R$ package (Oksanen et al., 2018). As a post-hoc test we carry out a Wilcoxon

(ii) leaf rate, or leaf production rate, calculated as the number of leaves derived from a single individual every week during four months of in vitro culture, or the slope of the linear regression model using the $l m$ function in $R$ (no. leaves $V s$. time) $\left(\mathrm{W}_{\mathrm{T}} \mathrm{N}=75, \mathrm{~W}\right.$ $\left.\mathrm{N}=75, \mathrm{D} \mathrm{N}=75, \mathrm{D}_{\mathrm{T}}=75\right)$;

(iii) height rate, or height increase rate, calculated as the height $(\mathrm{cm})$ of a single individual measured every week during four months of in vitro culture or the slope of the linear regression model using the $l m$ function in $R$ ( $\mathrm{cm} V s$. time) $\left(\mathrm{W}_{\mathrm{T}} \mathrm{N}=75, \mathrm{~W}\right.$ $\mathrm{N}=75, \mathrm{D} \mathrm{N}=75, \mathrm{D}_{\mathrm{T}}=75$;

(iv) microbial growth, measured as observable growth of either bacterial or fungal organisms associated to plant tissue, potentially attributable to possible endophyte overgrowth $\left(\mathrm{W}_{\mathrm{T}} \mathrm{N}=75, \mathrm{~W} N=75, \mathrm{D} N=75, \mathrm{D}_{\mathrm{T}}=75\right)$. In accordance with Quambusch \& Winkelmann (2018), we only considered as possible endophytes those microorganisms growing directly on the explant, not in the culture media;

(v) root development, determined by the average number of days that it took for the roots to develop after in vitro establishment, multiplied by the number of individuals that developed roots, and divided by the total number of analyzed individuals $\left(\mathrm{W}_{\mathrm{T}}\right.$ $\mathrm{N}=75, \mathrm{~W} \mathrm{~N}=75, \mathrm{D} \mathrm{N}=75, \mathrm{D}_{\mathrm{T}}=75$ ).

No transformation of the dataset was carried out for further analysis.

\section{Data analysis} 225 simultaneously, we carry out a Permutational Multivariate Analysis of Variance 
228 rank sums test in order to distinguish differences in the individual traits. This statistical approach 229 was performed based on Rebollar et al., (2017). In addition, to evaluate the potential differences 230 between the analyzed populations, we conducted a Non-Metric Multidimensional Scaling 231 (NMDS) with Manhattan distance in the vegan $R$ package (Oksanen et al., 2018). We selected 232 the NMDS due to its flexibility, which allowed us to use different types of variables and make 233 few assumptions about the nature of the data (Legendre \& Legendre, 2012). Given that the 234 number of entries for "propagation rate" was higher than for the rest of the evaluated traits, we 235 subsampled these entries with the "sample" function in $R$. To evaluate if the subsample dataset 236 was representative of the full dataset, we conducted a paired sample T-test. All the analyses were 237 conducted using $R$ software (version 2.4-6) (R Core Team, 2013). 


\section{Results}

239 In vitro culture performance is significantly different between $W$ and $W_{T}$ populations

240 The analysis for the in vitro culture performance traits in wild populations with $\left(\mathrm{W}_{\mathrm{T}}\right)$ and without

241 (W) transgenes shows statistically significant differences between populations for all traits

242 (PERMANOVA, $F=7.81, p=0.0009$ ) and for three out of the five individual traits according to

243 the Wilcoxon test ("height rate" $p=4.95 \mathrm{e}-12$; "microbial growth" $p=8.3 \mathrm{e}-06$; "propagation rate";

$244 p=0.006)$. Figure $1(\mathrm{~A}-\mathrm{E})$ shows the values for all analyzed traits per population. In particular, we

245 want to emphasize that "height rate" as an in vitro performance trait had the largest difference

246 between $\mathrm{W}$ and $\mathrm{W}_{\mathrm{T}}$ populations.

247 Multivariate analysis of in vitro performance traits (NMDS) in $\mathrm{W}$ and $\mathrm{W}_{\mathrm{T}}$ populations (Fig. 1F) 248 shows different phenotypic variations attributable to each population $\left(\mathrm{W}\right.$ and $\left.\mathrm{W}_{\mathrm{T}}\right)$. "Propagation 249 rate" is a trait positively related to W population; in contrast, "microbial growth" is positively 250 related to $\mathrm{W}_{\mathrm{T}}$ population.

251

252

In vitro culture performance differs between $W$ and $D$ populations

253 The analysis for the in vitro culture performance traits in wild (W) and domesticated (D) 254 populations does show statistically significant differences between populations for all traits 255 (PERMANOVA, $F=9.43, p=0.0009$ ), and for four out of the five individual traits: "leaf rate" 256 (Wilcoxon, $p=9.74 \mathrm{e}-05$ ), "propagation rate" (Wilcoxon, $p=0.002$ ), "root development" 257 (Wilcoxon $p=0.001$ ), and "height rate" (Wilcoxon $p=2.52 \mathrm{e}-11$ ) (Fig. 2A-E).

258 Moreover, although the graphic representation of multivariate analysis of in vitro performance 259 traits (NMDS) in W and D populations shows overlapping of the ellipses representing each 
260 population, the statistical analysis of multivariate differences (PERMANOVA) in both 261 populations is statistically significant (Fig. 2F).

262

263

In vitro culture performance differs between $W_{T}$ and $D_{T}$ populations and between $D$ and $D_{T}$

264 populations

265 The analysis for in vitro culture performance traits in wild $\left(\mathrm{W}_{\mathrm{T}}\right)$ and domesticated $\left(\mathrm{D}_{\mathrm{T}}\right)$ 266 populations with transgenes shows statistically significant differences between populations in 267 four out of the five in vitro performance traits (Wilcoxon "height rate" $p=0.008$; "leaf rate" $p=$ 4.47e-05; "propagation rate" $p=0.02$; "root development" $p=0.02$ ) (PERMANOVA, $F=5.62, p=$ 0.002). Figure 3 (A-E) shows the values for all the analyzed traits per population. In particular, we want to emphasize that although $\mathrm{W}_{\mathrm{T}}$ has higher values for "root development", "leaf rate" 271 and "height rate" traits, $\mathrm{D}_{\mathrm{T}}$ population has higher "propagation rate".

272 In the case of the analysis of domesticated populations with $\left(\mathrm{D}_{\mathrm{T}}\right)$ and without $(\mathrm{D})$ transgenes, we 273 also found statistically significant differences in three out of the five analyzed traits (Wilcoxon 274 "microbial growth" $p=0.001$; "propagation rate" $p=0.03$; "root development" $p=0.04$; )

275 (PERMANOVA, $F=3.86, p=0.0008$ ) (Fig. 3G-K), showing that $\mathrm{D}_{\mathrm{T}}$ in general has a better in 276 vitro performance.

277 The multivariate analysis of in vitro performance traits $(\mathrm{NMDS})$ in $\mathrm{W}_{\mathrm{T}}$ and $\mathrm{D}_{\mathrm{T}}$ populations (Fig. $2783 \mathrm{~F})$ shows different phenotypic variations attributable to each population $\left(\mathrm{W}_{\mathrm{T}}\right.$ and $\left.\mathrm{D}_{\mathrm{T}}\right)$, which 279 coincides with the same analysis for W and D populations (with no transgenes identified; Fig. 280 2F). Moreover, "propagation rate" is positively related to $\mathrm{D}_{\mathrm{T}}$ population, while the rest of the 281 traits seems positively related to $\mathrm{W}_{\mathrm{T}}$ population. 
282 In the analysis of $\mathrm{D}$ and $\mathrm{D}_{\mathrm{T}}$ populations, the NMDS (Fig. 3L) shows overlapping of the ellipses

283 representing the data set distribution of both populations, despite the significance of the 284 statistical analysis (PERMANOVA).

285 To look at the full data set $\left(\mathrm{W}, \mathrm{W}_{\mathrm{T}}, \mathrm{D}, \mathrm{D}_{\mathrm{T}}\right)$, and beyond the pairwise hypotheses, we carried out 286 the NMDS and PERMANOVA analyses. The results of the full data set analyses are coherent 287 with the pairwise comparisons, which supports the above mentioned observations (Fig. S5).

\section{Discussion}

289 Conservation of the genetic and phenotypic diversity in the CWR has been acknowledged as key 290 in the preservation of diverse gene pools to secure genetic alternatives for future decisions and 291 interventions regarding crop production. Of the different conservation strategies that exist, in 292 vitro gene banks represent a robust approach to preserve genetic diversity without introducing 293 unintended variations into the genetic pool (Engelmann, 1991; Gosal \& Kang, 2012). In recent 294 decades, the use of GMOs has become extensive (ISAAA, 2017), and a source of new genetic 295 variation, even in centers of origin of important crops ( $\mathrm{Lu}, 2008)$. This makes it important to 296 evaluate evidence of the effects, if there are any, of this introduced variation on in vitro culture 297 germplasm conservation efforts.

298

In order to analyze evidence of potential effects of transgene presence in cotton 300 metapopulations, we compared in vitro performance traits in metapopulations with $\left(\mathrm{W}_{\mathrm{T}}\right)$ and 301 without (W) transgenes. We found significant differences in in vitro culture performance 302 between $\mathrm{W}$ and $\mathrm{W}_{\mathrm{T}}$ populations for three out of the five analyzed traits (Fig. 1A-E). Previous studies with different crop populations have shown that even small genotypic changes can have major impact in phenotypes and fitness traits both in field experimental settings (Hernández- 
305 Terán et al., 2017) and in in vitro culture (Gandonou et al., 2005; Landi \& Mezzetti, 2006;

306 Kumar \& Reddy, 2011). Thus, it can be argued that the observed differences in in vitro culture

307 performance could be the result of natural genetic variation within and among populations,

308 however when we look at potential differences among $\mathrm{W}$ metapopulations, we found no

309 statistically significant differences (Supplementary Material Appendix S4). Nonetheless, the

310 observed differences between $\mathrm{W}_{\mathrm{T}}$ and $\mathrm{W}$ populations could be attributed, as in other studies, to

311 pleiotropic effects, where certain phenotypic traits may be linked to and affected by the genetic

312 modification of another trait (Filipecki \& Malepszy, 2006). In this sense, some studies have

313 shown that genetic modification can alter metabolic pathways due to position effects of

314 transgenes or somaclonal variation during tissue culture (Agapito-Tenfen et al., 2013; Mesnage

315 et al., 2016). In the specific case of cotton (Wang et al., 2015), some authors have found

316 overexpression of metabolites related to energy metabolism pathways that could indicate an

317 increased demand for energy, and concomitant changes in resource allocation and development.

318 Pleiotropic effects, have also been attributed to bottlenecks, selective sweeps, phenotypic

319 plasticity or gene x environment (GxE) interactions (Remington et al., 2001; Pozzi et al., 2004;

320 Gunasekera et al., 2006; Doust et al., 2014). In addition, ecological costs in different plant

321 species have been associated with the expression of transgenes (Chen et al., 2006). Specifically,

322 some studies show that the physiological production of transgene toxins (e.g. Cry proteins) is

323 extremely costly, limiting the energy destined for growth and reproduction. This trade-off caused

324 by the genetic modification has been found in Brassica (Snow, Andersen \& Jorgensen, 1999)

325 and Arabidopsis (Bergelson et al., 1996). Although in our study we cannot attribute these

326 performance differences only to the presence of transgenes (i.e. no strict control of genotypes),

327 we can say that all individuals identified as $\mathrm{W}_{\mathrm{T}}$ were positive for the expression of transgene 
328 proteins in the ELISA approach, which is suggestive of a potential cost that gets reflected in the

329 in vitro performance of $\mathrm{W}_{\mathrm{T}}$ populations. In general, in vitro performance differences between $\mathrm{W}$

330 and $\mathrm{W}_{\mathrm{T}}$ could be explained, at least with the information here collected, by ecological costs

331 associated to the expression of transgenes and by potential pleiotropic and GxE interactions

332 associated with small genetic differences.

333

334

In order to isolate the effect of domestication from the effect of transgenes on the in vitro

performance of cotton populations, we compared the in vitro performance of both wild metapopulations and domesticated populations with and without transgenes. In the case of comparisons of populations without transgenes (W-D) we found significant differences in four out of the five analyzed traits (Fig. 2A-E). Such phenotypic differentiation, regardless of substantial evolutionary divergence and genetic differentiation (Fang et al., 2017), is somehow unexpected, since differentiation between these populations is the result of a selective process focused on traits that are not related to in vitro performance, such as length, size and color of the

342 fiber, loss of seed dispersal and germination speed (Lubbers \& Chee, 2009; Gross \& Strasburg, 343 2010; Velázquez-López et al., 2018). Nonetheless, strong selective forces associated with

344 domestication and divergence times between populations are together of sufficient strength to 345 show phenotypic differentiation even in an environment to which both $\mathrm{W}$ and $\mathrm{D}$ populations 346 were naïve to (in vitro conditions). 
351 with the important exception of one trait, "propagation rate". This better performance of $\mathrm{D}_{T}$

352 populations for "propagation rate" could be the result of a history of selection in in vitro culture,

353 which is part of the conventional process of genetic engineering, through which transgenes are

354 introduced in the domesticated plants' genetic backgrounds (Hooykaas \& Schilperoort, 1992).

355 This suggests that since GMOs have previously gone through an in vitro process, it could be

356 possible that GM plants that have been selected for culture are better adapted to these conditions.

357 In contrast, for the rest of traits, $\mathrm{W}_{\mathrm{T}}$ populations perform better (higher "height rate", "leaf rate",

358 and "root development"), to which a potential mechanistic explanations or hypotheses are hard to

359 articulate. One possibility is that, given the reduced genetic variation of D populations in

360 comparison with $\mathrm{W}$ populations, the general performance of $\mathrm{D}$ populations might be expected to

361 be worse in environmentally astringent conditions (Flint-garcia, 2013; Lu, 2013), such as in vitro

362 culture.

363 Regardless of the trait performance direction of populations $\left(\mathrm{W}_{\mathrm{T}}\right.$ and $\left.\mathrm{D}_{\mathrm{T}}\right)$, we can argue 364 that given the existent differences between D and W genotypes without transgenes (see above 365 and (Velázquez-López et al., 2018)), it is expected that additional genetic changes (due to gene 366 insertion) could contribute to increased phenotypic differentiation. Nonetheless, given that we

367 did not determine the exact location of the inserted transgenes in the genome, it is not possible to 368 give a mechanistic explanation to the specific trait differences. Overall, we can conclude that our 369 results suggest that the presence of transgenes, originally associated with domesticated 370 populations, has a significant impact on the in vitro performance of the genotypes, regardless of 371 their wild or domesticated origin.

372

373 Implications of transgene presence for in vitro wild germplasm conservation 
374 One of the best ex situ conservation strategies for wild germplasm are in vitro banks (Gosal \&

375 Kang, 2012). In vitro conservation success depends on efficient and reliable micropropagation or

376 in vitro performance of the species of interest (Mycock, Blakeway \& Watt, 2004). Despite the

377 reality of crop intensification, including genetic engineering, the possible consequences of the

378 presence of transgenes for the in vitro performance of populations are poorly documented. In this

379 study, we present results that suggest detrimental consequences for the in vitro culture

380 performance of wild cotton populations in the presence of transgenes, which calls for monitoring

381 transgenes in the plants to be micropropagated for conservation or future genetic improvement,

382 as has been suggested by other authors, as conservation strategies and protocols (Bhatia, 2015).

383 Moreover, it is worth noting that in the present study, with a minimal investment of three primer

384 sets for transgene detection, we were able to identify 23 out of 33 transformation events reported

385 for cotton populations in Mexico (ISAAA, 2018). As it stands, our results provide experimental

386 evidence to support the implementation of transgene screening of plants to reduce time and

387 economic costs in in vitro establishment, helping the overarching goal of germplasm

388 conservation for future adaptation.

389 In current scenarios of global change, uncertain future conditions pose the major

390 challenge of securing resources for future adaptations (Wise et al., 2014). In this sense, it is of

391 utmost importance to preserve options for future decisions and to guarantee the right to

392 biodiversity and cultural identity for future generations, which includes genetic and phenotypic

393 options (Rockstrom et al., 2014). Crop biodiversity preservation is, in other words, part of our

394 life insurance for future adaptation in a changing planet. In this sense, future work on

395 conservation strategies and policies should put effort in expanding the knowledge about the

396 consequences of transgene presence (Lu, 2013) beyond the immediate gene pool of wild 
397 populations. This means extending the efforts breadth towards other interfertile species; in other 398 words, to the genetic primary pool.

399

400 Conclusions

401 The results presented show how transgene presence in CWR cotton populations has negative 402 consequences for their in vitro culture performance. In particular, reviewing our hypotheses, we 403 found that (1) in vitro culture performance is significantly different between $\mathrm{W}$ and $\mathrm{W}_{\mathrm{T}}$ 404 populations, and (2) in vitro culture performance is different between wild and domesticated 405 populations regardless of transgene presence. Overall, our results suggest that the presence of 406 transgenes in wild populations imposes a cost (e.g. metabolic cost of maintaining the expression 407 of toxins) that is reflected in their in vitro performance and that could endanger the success of 408 germplasm conservation efforts. Further studies controlling for specific genotypes and specific 409 transgene constructions would help to better disentangle the costs associated with specific 410 genomic contexts and genetic modifications to improve genetic screenings for in vitro banks.

\section{Acknowledgments}

413 The authors would like to thank Morena Avitia, Lidia Cabrera, Andrea Jiménez, Luis Barba 414 Escoto, and Joel Reyna for technical assistance. The authors acknowledge Dra. Florencia García415 Campusano for her support in the laboratory work, and to the Laboratorio de Biotecnología 416 CENID-COMEF, INIFAP.

\section{Supporting information}


419 Specifics of the in vitro culture used in the establishment and propagation of the germplasm

420 collection (Appendix S1), a detailed scheme of the experimental design (Appendix S2), primers

421 sequences used for transgene detection (Appendix S3), multivariate analysis (NMDS) result of

422 the metapopulation comparison (Appendix S4), integrated analysis (NMDS) of all genotypes

423 (Appendix S5), and raw sequences of transgene detection (Appendix S6) are available online.

424

$425 \quad$ Literature cited

426 Agapito-Tenfen SZ, Guerra MP, Wikmark OG, Nodari RO. 2013. Comparative proteomic

427 analysis of genetically modified maize grown under different agroecosystems conditions in

428 Brazil. Proteome Science 11. DOI: 10.1186/1477-5956-11-46.

429 Bergelson J, Purrington CB, Palm CJ, López-Gutiérrez JC. 1996. Costs of resistance: A test

430 using transgenic Arabidopsis thaliana. Biological Sciences 263:1659-1663. DOI:

$431 \quad 10.1098 / \mathrm{rspb} .1996 .0242$.

432 Bhatia S. 2015. Application of plant biotechnology. In: Modern Applications of Plant

433 Biotechnology in Pharmaceutical Sciences. Elsevier Inc., 157-207. DOI: 10.1016/B978-0-

$434 \quad 12-802221-4.00005-4$.

435 Burgeff C, Huerta E, Acevedo F, Sarukhán J. 2014. How much can GMO and Non-GMO

436 cultivars coexist in a megadiverse country? AgBioForum 17:90-101.

437 Castañeda-Álvarez NP, Khoury CK, Achicanoy HA, Bernau V, Dempewolf H, Eastwood RJ,

438 Guarino L, Harker RH, Jarvis A, Maxted N, Müller J V., Ramirez-Villegas J, Sosa CC,

439 Struik PC, Vincent H, Toll J. 2016. Global conservation priorities for crop wild relatives.

$440 \quad$ Nature Plants 2:1-6. DOI: 10.1038/NPLANTS.2016.22.

441 Chen L-Y, Snow AA, Wang F, Lu B. 2006. Effects of insect-resistance transgenes on fecundity 
442

443

444

445

446

447

448

449

450

451

452

453

454

455

456

457

458

459

460

461

462

463

464

in rice (Oryza sativa, Poacea): a test for underlying costs. American Journal of Botany 93:94-101. DOI: 10.3732/ajb.93.1.94.

Doust AN, Lukens L, Olsen KM, Mauro-Herrera M, Meyer A, Rogers K. 2014. Beyond the single gene: how epistasis and gene-by-environment effects influence crop domestication. Proceedings of the National Academy of Sciences 111:6178-6183. DOI: $10.1073 /$ pnas. 1308940110.

Ellstrand NC. 2018. "Born to Run"? Not Necessarily: Species and Trait Bias in Persistent FreeLiving Transgenic Plants. Frontiers in Bioengineering and Biotechnology 6:1-10. DOI: 10.3389/fbioe. 2018.00088 .

Engelmann F. 1991. In vitro conservation of tropical plant germplasm -a review. Euphytica 57:227-243. DOI: 10.1007/BF00039669.

Fang L, Gong H, Hu Y, Liu C, Zhou B, Huang T, Wang Y, Chen S, Fang DD, Du X, Chen H, Chen J, Wang S, Wang Q, Wan Q, Liu B, Pan M, Chang L, Wu H, Mei G, Xiang D, Li X, Cai C, Zhu X, Chen ZJ, Han B, Chen X, Guo W, Zhang T, Huang X. 2017. Genomic insights into divergence and dual domestication of cultivated allotetraploid cottons. Genome Biology 18:1-13. DOI: 10.1186/s13059-017-1167-5.

Filipecki M, Malepszy S. 2006. Unintended consequences of plant transformation: a molecular insight. Journal of applied genetic 47:277-286. DOI: 10.1007/BF03194637.

Flint-garcia SA. 2013. Genetics and consequences of crop domestication. Journal of Agricultural and Food Chemistry 61:8267-8276. DOI: 10.1021/jf305531j.

Gandonou C, Errabii T, Abrini J, Idaomar M, Chibi F, Skali Senhaji N. 2005. Effect of genotype on callus induction and plant regeneration from leaf explants of sugarcane (Saccharum sp.). African Journal of Biotechnology 4:1250-1255. DOI: 10.4314/ajb.v4i11.71384. 
465 Gosal SS, Kang MS. 2012. Plant tissue culture and genetic transformation for crop improvement.

466 In: Tuteja N, Gill SS, Tiburcio AF, Tuteja R eds. Improving crop resistance to abiotic

467 stress. Wiley-VCH, 253-279. DOI: 10.1016/B978-0-08-091753-5.50013-5.

468 Greene SL, Kesoju SR, Martin RC, Kramer M. 2015. Occurrence of transgenic feral alfalfa

469 (Medicago sativa subsp. sativa L.) in alfalfa seed production areas in the United States.

$470 \quad$ PLoS ONE 10:1-17. DOI: 10.1371/journal.pone.0143296.

471 Gross BL, Strasburg JL. 2010. Cotton domestication: dramatic changes in a single cell. BMC

472 biology 8:137. DOI: 10.1186/1741-7007-8-137.

473 Gubis J, Lajchova Z, Farago J, Jurekova Z. 2003. Effect of genotype and explant type on shoot

474 regeneration in tomato (Lycopersicon esculentum Mill.) in vitro. Czech Journal of Genetics

475 and Plan Breeding 39:9-14. DOI: 10.1104/pp.91.2.694.

476 Gunasekera CP, Martin LD, Siddique KHM, Walton GH. 2006. Genotype by environment

477 interactions of indian mustard (Brassica juncea L.) and canola (B. napus L.) in

478 mediterranean-type environments 1. Crop growth and seed yield. European Journal of

479 Agronomy 25:1-12. DOI: 10.1016/j.eja.2005.08.002.

480 Hajjar R, Hodgkin T. 2007. The use of wild relatives in crop improvement: a survey of

481 developments over the last 20 years. Euphytica 156:1-13. DOI: 10.1007/s10681-007-9363-

$482 \quad 0$.

483 Harlan JR. 1965. The possible role of weed races in the evolution of cultivated plants. Euphytica 484 14:173-176. DOI: 10.1007/BF00038984.

485 Hawkes JG. 1977. The importance of wild germplasm in plant breeding. Euphytica 26:615-621.

486 DOI: $10.1007 /$ BF00021686.

487 Hernández-Terán A, Wegier A, Benítez M, Lira R, Escalante AE. 2017. Domesticated, 
488

489

490

491

492

493

494

495

496

497

498

499

500

501

502

503

504

505

506

507

508

509

510

Genetically Engineered, and Wild Plant Relatives Exhibit Unintended Phenotypic

Differences: A Comparative Meta-Analysis Profiling Rice, Canola, Maize, Sunflower, and Pumpkin. Frontiers in Plant Science 8:1-10. DOI: 10.3389/fpls.2017.02030.

Heywood V, Casas A, Ford-Lloyd B, Kell S, Maxted N. 2007. Conservation and sustainable use of crop wild relatives. Agriculture, Ecosystems and Environment 121:245-255. DOI: 10.1016/j.agee.2006.12.014.

Hooykaas PJJ, Schilperoort RA. 1992. Agrobacterium and plant genetic engineering. Plant molecular biology 19:15-38. DOI: 10.1007/BF00015604.

Hunter D, Heywood V (eds.). 2011. Crop wild relatives: a manual of in situ conservation. New York, NY: Earthscan.

ISAAA. 2017. Global Status of Commercialized Biotech/GM Crops in 2017: Biotech Crop Adoption Surges as Economic Benefits Accumulate in 22 Years. Ithaca, NY.

ISAAA. 2018.GM approval database. Available at http://www.isaaa.org/gmapprovaldatabase/ (accessed November 5, 2018).

Kumar N, Reddy MP. 2010. Plant regeneration through the direct induction of shoot buds from petiole explants of Jatropha curcas: a biofuel plant. Annals of Applied Biology 156:367-375. DOI: $10.1111 / \mathrm{j} .1744-7348.2010 .00394 . x$.

Kumar N, Reddy MP. 2011. In vitro plant propagation: a review. Journal of Forest Science 27:61-72.

Landi L, Mezzetti B. 2006. TDZ, auxin and genotype effects on leaf organogenesis in Fragaria. Plant Cell Reports 25:281-288. DOI: 10.1007/s00299-005-0066-5.

Leadley PW, Krug CB, Alkemade R, Pereira HM, Sumaila UR, Walpole M, Marques A, Newbold T, Teh LSL, van Kolck J, Bellard C, Januchowski-Hartley SR, Mumby PJ. 2014. 
511

512

513

514

515

516

517

518

519

520

521

522

523

524

525

526

527

528

529

530

531

532

533

Progress towards the aichi biodiversity targets: An assessment of biodiversity trends, policy scenarios and key actions. Montreal, Canada: Secretariat of the Convention on Biological Diversity.

Legendre P, Anderson MJ. 1999. Distance-based redundancy analysis: testing multispecies reponses in multifactorial ecological experiments. Ecological Monographs 69:1-24. DOI: 10.1890/0012-9615(1999)069[0001:DBRATM]2.0.CO;2.

Legendre P, Legendre L. 2012. Ordination in reduced space. In: Numerical Ecology. Amsterdam, Netherlands: Elsevier, 1006.

Li W, Masilamany P, Kasha KJ, Pauls KP. 2002. Developmental, tissue culture, and genotypic factors affecting plant regeneration from shoot apical meristems of germinated Zea mays L. seedlings. In Vitro Cellular \& Developmental Biology - Plant 38:285-292. DOI: 10.1079/IVP2002291.

Lu B-R. 2008. Transgene escape from GM crops and potential biosafety consequences: an environmental perspective. Collection of Biosafety reviews 4:66-141.

Lu B-R. 2013. Introgression of transgenic crop alleles : Its evolutionary impacts on conserving genetic diversity of crop wild relatives. Journal of Systematics and Evolution 51:245-262. DOI: $10.1111 /$ jse. 12011 .

Lubbers EL, Chee PW. 2009. The worldwide gene pool of G. hirsutum and its improvement. In: Paterson AH ed. Genetics and genomics of cotton. New York, NY: Springer, 23-53. DOI: 10.1007/978-0-387-70810-2.

Manshardt R, Bishaw D, Pitz K, Stewart CN. 2016. Gene flow from commercial transgenic papaya fields into feral populations in Hawaii. Acta Horticulturae 1124:33-40. DOI: 10.17660/ActaHortic.2016.1124.5. 
534 Mesnage R, Agapito-Tenfen SZ, Vilperte V, Renney G, Ward M, Séralini GE, Nodari RO,

535 Antoniou MN. 2016. An integrated multi-omics analysis of the NK603 Roundup-tolerant

536 GM maize reveals metabolism disturbances caused by the transformation process. Scientific

537 Reports 6:1-14. DOI: 10.1038/srep37855.

538 Murashige T, Skoog F. 1962. A revised medium for rapid growth and bio assays with tobacco

539 tissue cultures. Physiologia Plantarum 15:473-497. DOI: 10.1111/j.1399-

$540 \quad$ 3054.1962.tb08052.x.

541 Mycock DJ, Blakeway FC, Watt MP. 2004. General applicability of in vitro storage technology

542 to the conservation and maintenance of plant germplasm. South African Journal of Botany

543 70:31-36. DOI: 10.1016/S0254-6299(15)30265-9.

544 Oksanen J, Blanchet GF, Friendly M, Kindt R, Legendre P, McGlinn D, Minchin PR, O’Hara

545 RB, Simpson GL, Solymos P, Stevens MHH, Szoecs E, Wagner H. 2018. Vegan:

$546 \quad$ community ecology package.

547 Pathi KM, Tuteja N. 2013. High-frequency regeneration via multiple shoot induction of an elite

548 recalcitrant cotton (Gossypium hirsutum L. cv Narashima) by using embryo apex. Plant

549 signaling \& behavior 8. DOI: 10.4161/psb.22763.

550 Piñeyro-Nelson A, Van Heerwaarden J, Perales HR, Serratos-Hernández JA, Rangel A, Hufford

551 MB, Gepts P, Garay-Arroyo A, Rivera-Bustamante R, Álvarez-Buylla ER. 2009.

552 Transgenes in mexican maize: Molecular evidence and methodological considerations for

553 GMO detection in landrace populations. Molecular Ecology 18:750-761. DOI:

$554 \quad$ 10.1111/j.1365-294X.2008.03993.x.

555 Plucknett DL, Smith NJ, Williams JT, Anishetty NM. 1983. Crop germplasm conservation and 556 developing countries. Science 220:163-169. DOI: 10.1126/science.220.4593.163. 
557 Pozzi C, Rossini L, Vecchietti A, Salamini F. 2004. Gene and genome changes during

558 domestication of cereals. In: Gupta PK, Varshney RK eds. Cereal Genomics. Netherlands:

$559 \quad$ Kluwer Academic Publishers, 165-198.

560 Quambusch M, Winkelmann T. 2018. Bacterial endophytes in plant tissue culture: mode of 561 action, detection, and control. In: Loyola-Vargas VM, Ochoa-Alejo N eds. Plant cell 562 protocols. New York, NY, 69-88.

563 Quist D, Chapela IH. 2001. Transgenic DNA introgressed into traditional maize landraces in 564 Oaxaca, Mexico. Nature 414:541-543. DOI: 10.1038/35107068.

565 R Core Team. 2013. R: a language and environment for statistical computing. DOI: ISBN 3566 900051-07-0.

567 Rajasekharan PE, Sahijram L. 2015. In vitro conservation of plant germplasm. In: Bahadur B, 568 Rajam M V, Leela S, V KK eds. Plant Biology and Biotechnology: Volume II: Plant 569 Genomics and Biotechnology. India: Springer India,. DOI: 10.1007/978-81-322-2283.

570 Rebollar EA, Sandoval-Castellanos E, Roessler K, Gaut BS, Alcaraz LD, Benítez M, Escalante 571 AE. 2017. Seasonal changes in a maize-based polyculture of central Mexico reshape the co572 occurrence networks of soil bacterial communities. Frontiers in Microbiology 8. DOI: $573 \quad 10.3389 /$ fmicb.2017.02478.

574 Remington DL, Thornsberry JM, Matsuoka Y, Wilson LM, Whitt SR, Doebley J, Kresovich S, 575 Goodman MM, Iv ESB. 2001. Structure of linkage disequilibrium and phenotypic 576 associations in the maize genome. Proceedings of the National Academy of Sciences 577 98:11479-11484. DOI: 10.1073/pnas.201394398.

578 Rockstrom J, Steffen WL, Noone K, Persson A, Chapin III FS. 2014. Planetary boundaries: 579 exploring the safe operating space for humanity. Ecology and Society 14:81-87. DOI: 
581 Snow AS, Andersen B, Jorgensen RB. 1999. Costs of transgenic herbicide resistance

582 introgressed from Brassica napus into weedy B. rapa. Molecular Ecology 8:605-615. DOI:

$583 \quad 10.1046 /$ j.1365-294x.1999.00596.x.

584 Tyagi RK, Yusuf A, Dua P, Agrawal A. 2004. In vitro plant regeneration and genotype

585 conservation of eight wild species of Curcuma. Biologia Plantarum 48:129-132. DOI:

$586 \quad 10.1023 /$ B:BIOP.0000024289.68669.ef.

587 Ulloa M, Stewart JM, Garcia-C. EA, Godoy-A. S, Gaytan-M. A, Acosta NS. 2006. Cotton

588 genetic resources in the western states of mexico: in situ conservation status and germplasm

589 collection for ex situ preservation. Genetic Resources and Crop Evolution 53:653-668.

590 DOI: $10.1007 / \mathrm{s} 10722-004-2988-0$.

591 Velázquez-López R, Wegier A, Alavez V, Pérez-López J, Vázquez-Barrios V, Arroyo-Lambaer

592 D, Ponce-Mendoza A, Kunin WE. 2018. The mating system of the wild-to-domesticated

593 complex of Gossypium hirsutum L . is mixed. Frontiers in Plant Science 9:1-14. DOI:

$594 \quad 10.3389 /$ fpls.2018.00574.

595 Wang L, Wang X, Jin X, Jia R, Huang Q, Tan Y, Guo A. 2015. Comparative proteomics of Bt-

596 transgenic and non-transgenic cotton leaves. Proteome Science 13:1-15. DOI:

$597 \quad 10.1186 / \mathrm{s} 12953-015-0071-8$.

598 Warwick SI, Légère A, Simard MJ, James T. 2008. Do escaped transgenes persist in nature? The 599 case of an herbicide resistance transgene in a weedy Brassica rapa population. Molecular 600 Ecology 17:1387-1395. DOI: 10.1111/j.1365-294X.2007.03567.x.

601 Wegier AL, Piñeyro-Nelson A, Alarcón J, Gálvez-Mariscal A, Álvarez-Buylla E, Piñero D. 602 2011. Recent long-distance transgene flow into wild populations conforms to historical 
603

604

605

606

607

608

609

610

611

612

613

614

615

616

617

618

619

620

621

622

623

624

625

626

627

628

patterns of gene flow in cotton (Gossypium hirsutum) at its centre of origin. Molecular

Ecology 20:4182-4194. DOI: 10.1111/j.1365-294X.2011.05258.x.

Wise RM, Fazey I, Stafford Smith M, Park SE, Eakin HC, Archer Van Garderen ERM, Campbell B. 2014. Reconceptualising adaptation to climate change as part of pathways of change and response. Global Environmental Change 28:325-336. DOI:

$$
\text { 10.1016/j.gloenvcha.2013.12.002. }
$$

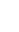

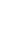

(n)

\section{Figure legends}

Figure 1. In vitro culture performance traits of $\mathbf{W}$ and $\mathbf{W}_{\mathrm{T}}$ populations. $\mathrm{W}$ : wild populations without transgenes, and $\mathrm{W}_{\mathrm{T}}$ : wild populations with transgenes. (A-E) median and standard error of all analyzed traits in both populations. $P$ values were obtained through Wilcoxon test. (F) Non-Metric Multidimensional Scaling (NMDS) that include all the analyzed traits in the two populations. The ellipses represent $95 \%$ confidence interval around the centroids.

Figure 2. In vitro culture performance traits of $\mathrm{W}$ and $\mathrm{D}$ populations. D: domesticated populations without transgenes, and $\mathrm{W}$ : wild populations without transgenes. (A-E) median and standard error of all analyzed traits in both populations. $P$ values were obtained through Wilcoxon test. (F) Non-Metric Multidimensional Scaling that include all the analyzed traits in the two populations. The ellipses represent $95 \%$ confidence interval around the centroids. 
629 Figure 3. In vitro culture performance traits of $D_{T}$ and $W_{T}$ and between $D$ and $D_{T}$ populations. In 630 genotype, $\mathrm{D}_{\mathrm{T}}$ : domesticated populations with transgenes, $\mathrm{W}_{\mathrm{T}}$ : wild populations with transgenes, D: 631 domesticated populations without transgenes. (A-E) (G-K) median and standard error of all analyzed traits in 632 both populations. $P$ values were obtained through Wilcoxon test. (F) (L) Non-Metric Multidimensional Scaling 633 that include all the analyzed traits in the analyzed populations. The ellipses represent $95 \%$ confidence interval 634 around the centroids.

635 


\section{Figure 1}

In vitro culture performance traits of $\mathrm{W}$ and $\mathrm{W}_{\mathrm{T}}$ populations

W: wild populations without transgenes, and $\mathrm{W}_{\mathrm{T}}$ : wild populations with transgenes. $(\mathrm{A}-\mathrm{E})$ median and standard error of all analyzed traits in both populations. $P$ values were obtained through Wilcoxon test. (F) Non-Metric Multidimensional Scaling (NMDS) that include all the analyzed traits in the two populations. The ellipses represent $95 \%$ confidence interval around the centroids
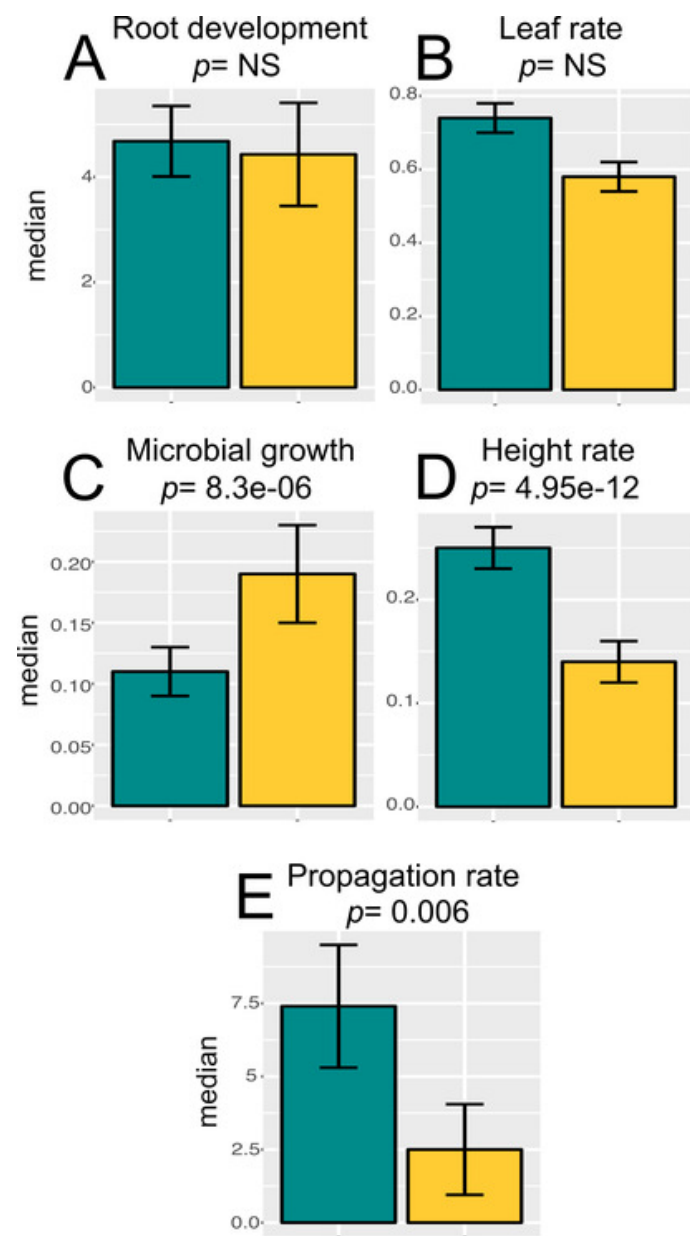

\section{F $\quad$ Genotype $W \bigcirc W_{T} \bigcirc$}

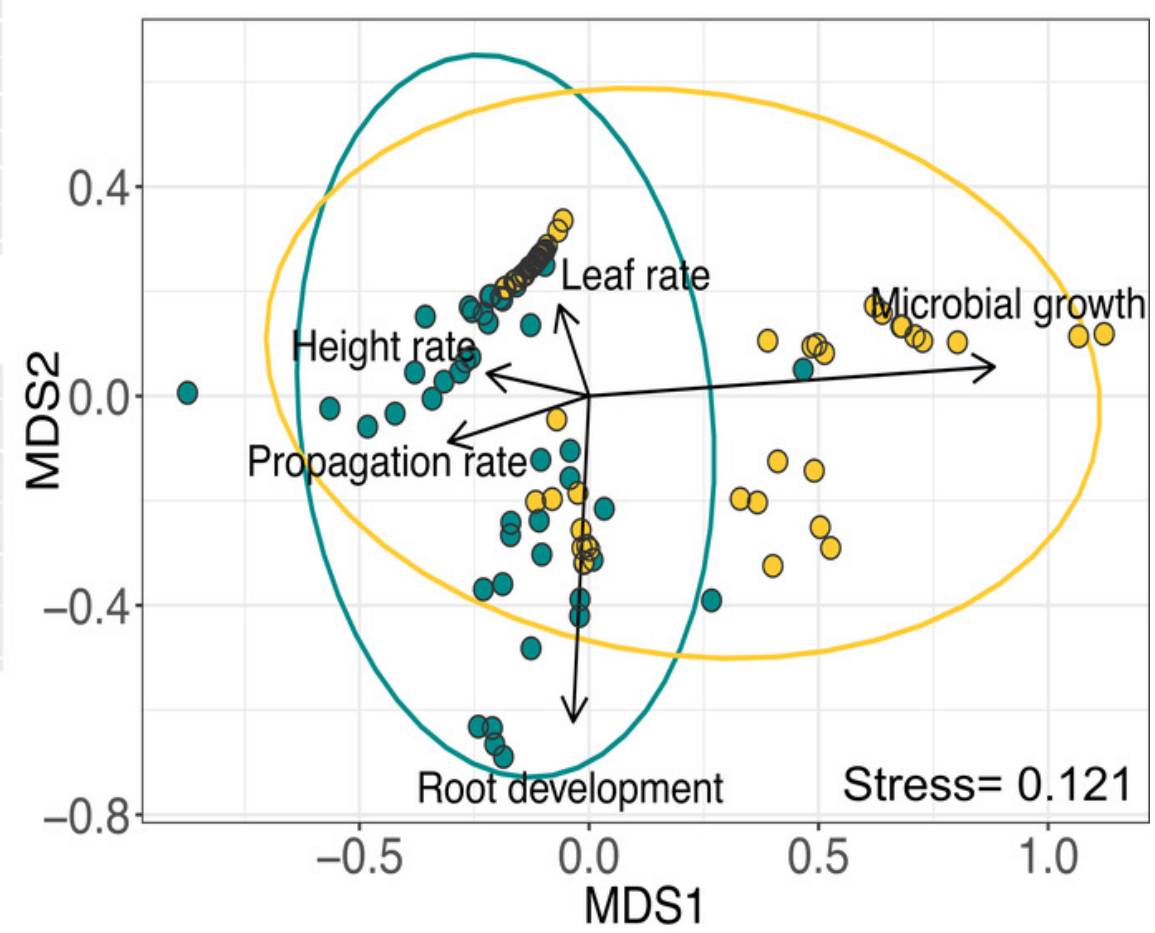

PERMANOVA $F=7.81, p=0.009$ 


\section{Figure 2}

In vitro culture performance traits of $\mathrm{W}$ and $\mathrm{D}$ populations

D: domesticated populations without transgenes, and W: wild populations without transgenes. (A-E) median and standard error of all analyzed traits in both populations. $P$ values were obtained through Wilcoxon test. (F) Non-Metric Multidimensional Scaling that include all the analyzed traits in the two populations. The ellipses represent $95 \%$ confidence interval around the centroids
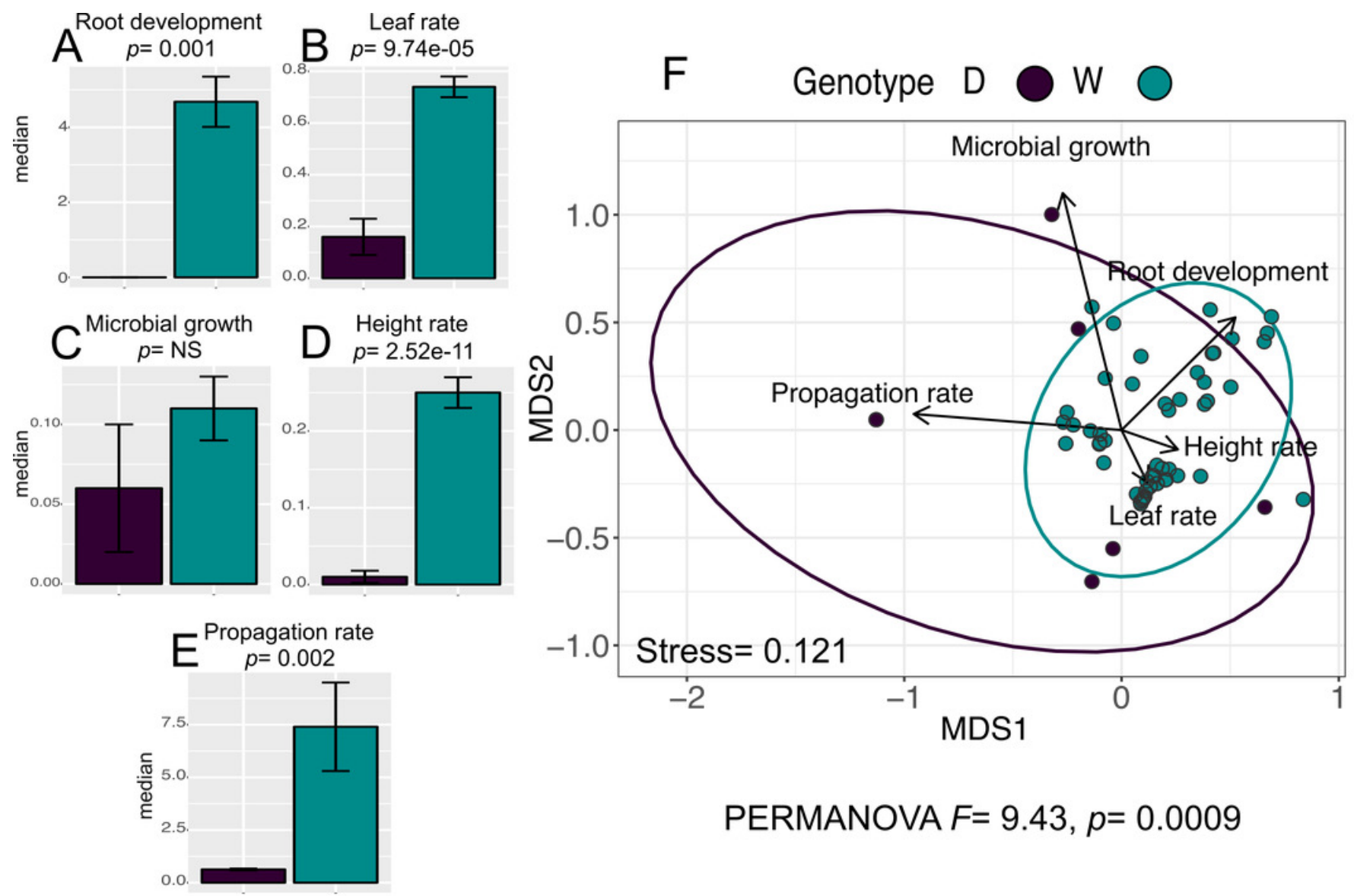

PERMANOVA $F=9.43, p=0.0009$ 


\section{Figure 3}

In vitro culture performance traits of $D_{T}$ and $W_{T}$ and between $D$ and $D_{T}$ populations

In genotype, $\mathrm{D}_{\mathrm{T}}$ : domesticated populations with transgenes, $\mathrm{W}_{\mathrm{T}}$ : wild populations with transgenes, D: domesticated populations without transgenes. (A-E) (G-K) median and standard error of all analyzed traits in both populations. $P$ values were obtained through Wilcoxon test. (F) (L) Non-Metric Multidimensional Scaling that include all the analyzed traits in the analyzed populations. The ellipses represent 95\% confidence interval around the centroids 
$\mathrm{A}^{\text {Root development }}$

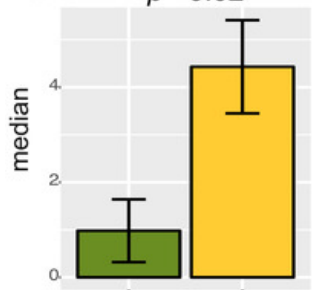

C Microbial growth
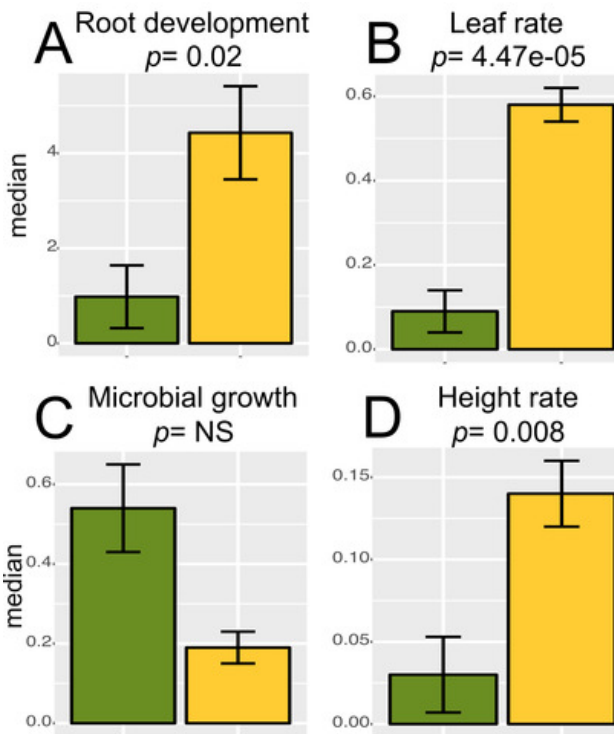

$\mathrm{D}$

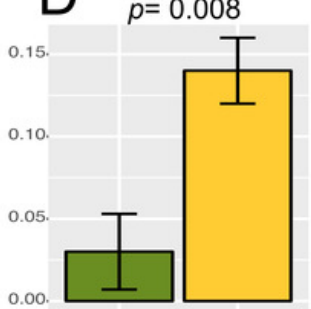

E Propagation rate
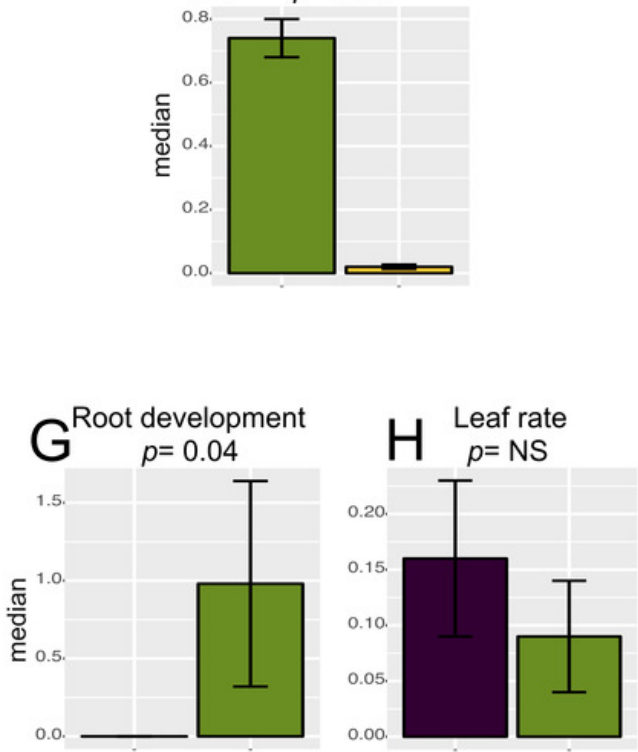

Microbial growth
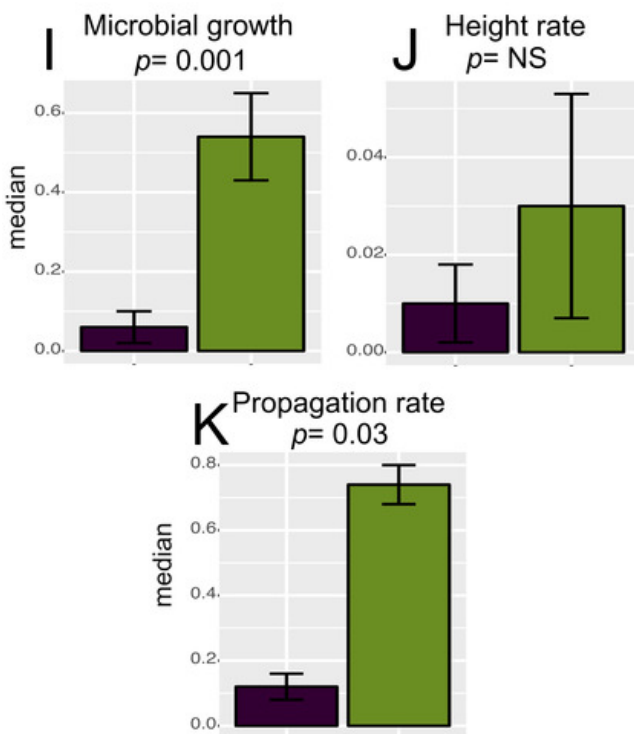

$F \quad$ Genotype $D_{T} \bigcirc W_{T} \bigcirc$

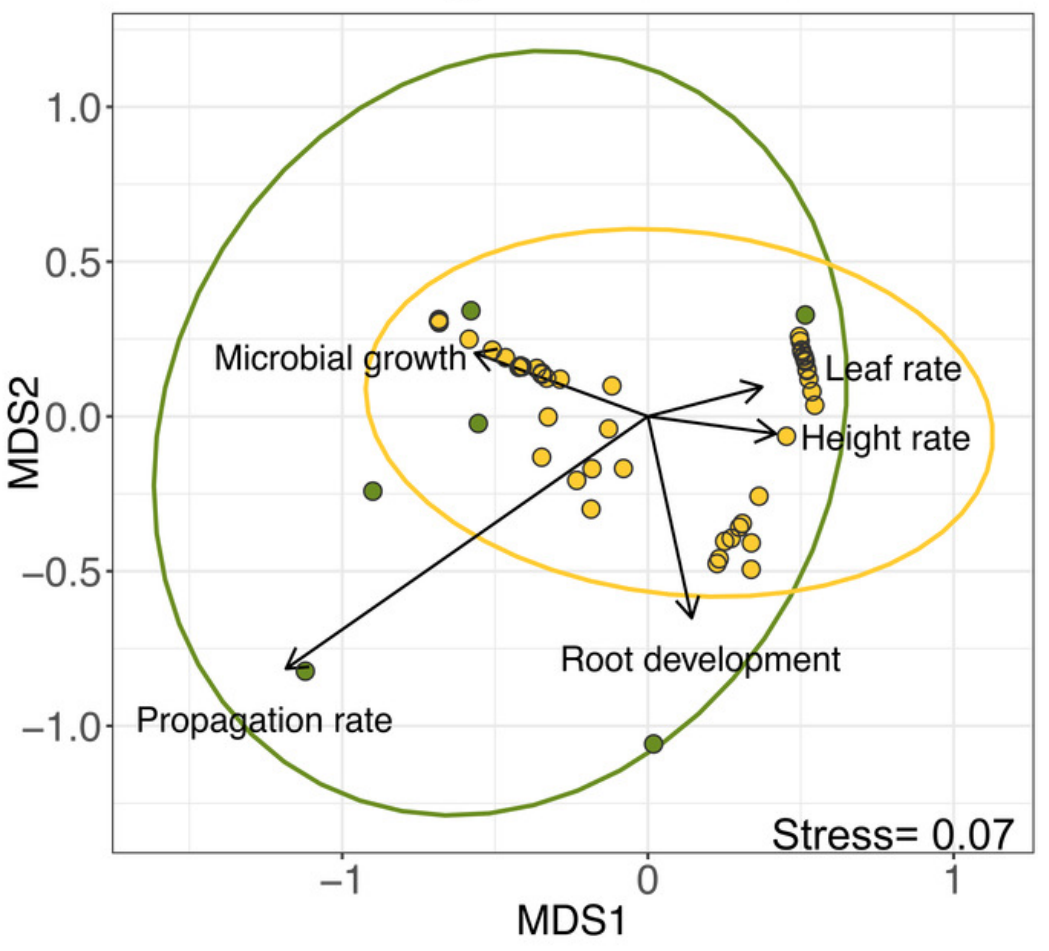

PERMANOVA $F=5.62, p=0.002$

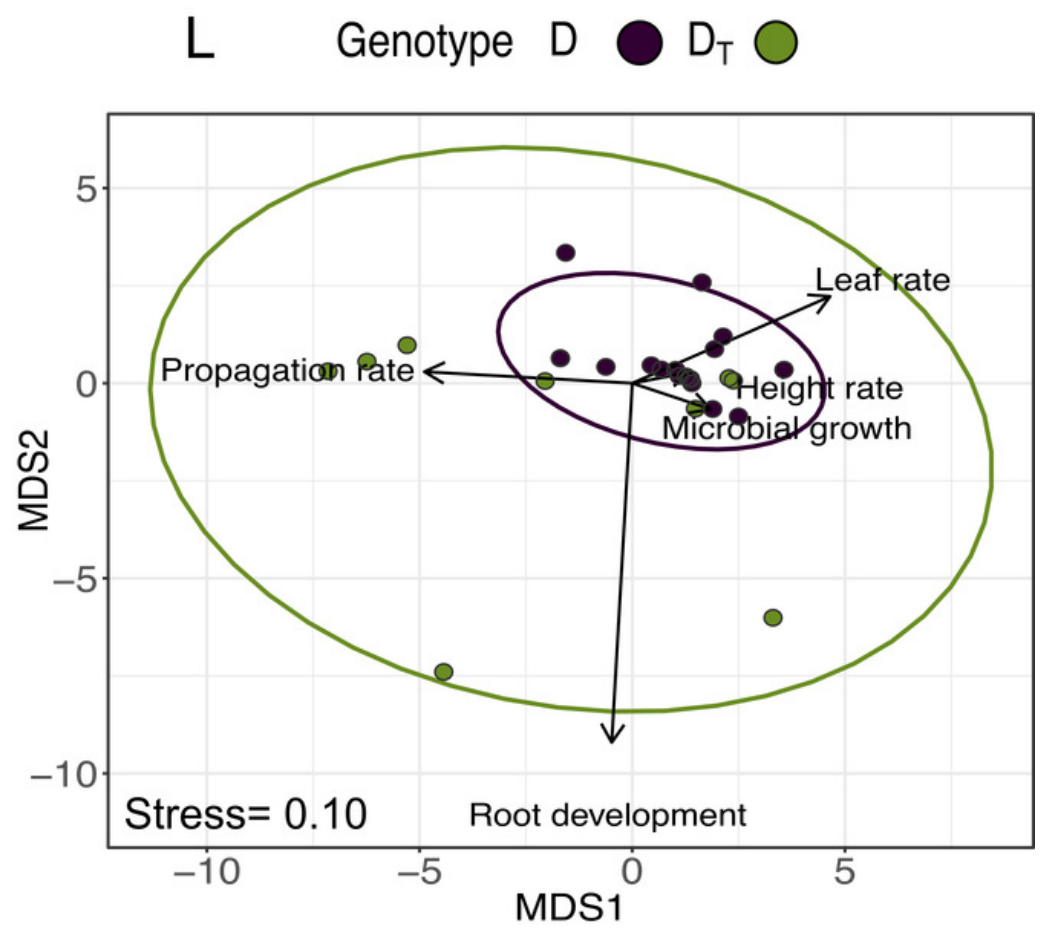

PERMANOVA $F=3.86, p=0.008$ 